\title{
The ATLAS Level-1 topological processor: experience and upgrade plans
}

\section{Paula Martínez Suárez* on behalf of the ATLAS Collaboration}

Institut de Física d'Altes Energies (IFAE-BIST), Universitat Autònoma de Barcelona (UAB) 08193, Bellaterra (Barcelona), Spain

E-mail: paula.martinez.suarez@cern.ch

During Run 2 the Large Hadron Collider has provided, at the World's energy frontier, protonproton collisions to the ATLAS experiment with high instantaneous luminosities of up to $2.1 \times 10^{34}$ $\mathrm{cm}^{2} \mathrm{~s}^{-1}$, placing stringent operational and physical requirements on the ATLAS trigger system in order to reduce the $40 \mathrm{MHz}$ collision rate to a manageable event storage rate of $1 \mathrm{kHz}$, while not rejecting interesting collisions. The Level-1 trigger is the first rate-reducing step in the ATLAS trigger system with an output rate of up to $100 \mathrm{kHz}$ and decision latency of less than 2.5 microseconds. Since 2017, an important role is played by the Level-1 topological processor. This innovative system consists of two blades designed in AdvancedTCA form factor, mounting four individual state-of-the-art processors, and providing high input bandwidth and low latency data processing.Up to 128 topological trigger algorithms can be implemented to select interesting events by applying kinematic and angular requirements on electromagnetic clusters, hadronic jets, muons and total energy reconstructed in the ATLAS apparatus. This resulted in a significantly improved background event rejection rate and improved acceptance of physics signal events, despite the increasing luminosity. The L1Topo system has become more and more important for physics analyses making use of low energy objects, commonly present in the heavy flavour or Higgs physics events for example. An overview of the L1Topo architecture, simulation and performance results during Run- 2 is presented alongside with upgrade plans for the L1Topo system to be installed for the future data taking that will start in 2022.

The Ninth Annual Conference on Large Hadron Collider Physics - LHCP2021

7-12 June 2021

Online

${ }^{*}$ Speaker 


\section{The ATLAS Level-1 topological processor}

The Large Hadron Collider (LHC) has provided, during Run 2, proton-proton collisions to the ATLAS detector [1] at a rate of $40 \mathrm{MHz}$. The Level-1 (L1) trigger is the first stage of the ATLAS trigger system, and performs hardware-based online event selection in order to reduce the collision rate down to $100 \mathrm{kHz}$, which is the maximum detector readout rate. The $\mathrm{L} 1$ trigger is constituted by the L1 Calorimeter trigger (L1Calo) and the L1 Muon trigger (L1Muon) systems, in combination with the Central Trigger Processor (CTP). In addition to this, the L1 topological processor (L1Topo) is introduced in order to perform calculations of topological quantities combining different trigger objects (TOBs) from L1Calo and L1Muon, also sending this information to the CTP.

\section{L1Topo architecture}

The L1Topo system consists of two AdvancedTCA-compliant modules, each of them equipped with two processor FPGAs (Xilinx Virtex7) to run the algorithms and one controller FPGA (Kintex7) to communicate with the external trigger systems. Each processor FPGA can hold up to 32 topological algorithms, and by the end of 2018, a total of 113 topological algorithms were implemented, validated and operated. The total latency of the L1Topo system is around $200 \mathrm{~ns}$, equivalent to 8 LHC bunch crossings.

\section{L1Topo algorithms}

During the collisions, the input data from each event is arranged in TOBs and sent to the L1Topo system. These TOBs are bit arrays containing $(\eta, \phi)$ location ${ }^{1}$, transverse momentum and other additional information particular to each TOB. L1Topo can receive up to 120 EM TOBs, 120 tau TOBs, 32 muon TOBs, 64 jet TOBs and 1 missing transverse momentum TOB.

The signals are sent to the processor FPGAs to execute the topological algorithms. These algorithms are able to compute $\Delta \eta, \Delta \phi, \Delta R$ and compare $(\eta, \phi)$ positions, calculate $m_{\text {inv }}, m_{\mathrm{T}}$ and $H_{\mathrm{T}}$, compute energy ratios and perform large- $\mathrm{R}$ jet clustering, among other things. In order to reduce the number of combinations when applying the topological algorithms, L1Topo also has a set of sort/select algorithms. The first type sorts the input objects by $p_{\mathrm{T}}$ and selects up to 6 leading objects of the list, while the second retrieves the first 10 objects above a particular $p_{\mathrm{T}}$ threshold without any kind of sorting. The resulting bits produced at the end of the execution of the topological algorithms are sent to the CTP.

\section{Trigger performance during Run 2}

Plenty of physics studies benefit from the topological algorithms [2]. For instance, in order to identify a $J / \psi$ meson decaying to muon pairs, non-topological triggers require two muons with low

\footnotetext{
${ }^{1}$ ATLAS uses a right-handed coordinate system with its origin at the nominal interaction point (IP) in the centre of the detector and the $z$-axis along the beam pipe. The $x$-axis points from the IP to the centre of the LHC ring, and the $y$-axis points upwards. Cylindrical coordinates $(r, \phi)$ are used in the transverse plane, $\phi$ being the azimuthal angle around the $z$-axis. The pseudorapidity is defined in terms of the polar angle $\theta$ as $\eta=-\ln \tan (\theta / 2)$. Angular distance is defined as $\Delta R=\sqrt{(\Delta \eta)^{2}+(\Delta \phi)^{2}}$.
} 
$p_{\mathrm{T}}$ (at least $6 \mathrm{GeV}$ ). Topological requirements like a muon pair invariant mass in a given range (2 $\left.\mathrm{GeV}<m_{\mu \mu}<9 \mathrm{GeV}\right)$ and a given angular separation $\left(0.2<\Delta R_{\mu \mu}<1.5\right)$ reduce the di-muon L1 trigger rate by up to a factor of 4 while largely preserving the signal acceptance.

Topological algorithms are also useful in B-physics, in particular in the test of lepton-flavour universality through the decay $B^{0} \rightarrow K^{* 0} e^{+} e^{-}$, where the two electrons are collimated and have low energy. This signature is particularly challenging due to the high background rate, so a combination of topological algorithms is used. The first one triggers on two electrons, each of them contained in a region of size $\Delta \eta \times \Delta \phi=0.2 \times 0.2$; the second one applies to the cases where these two electrons are very collimated and overlap, and the resulting object does not fit in this $\Delta \eta \times \Delta \phi=0.2 \times 0.2$ window. In this situation, a larger object (a $\Delta \eta \times \Delta \phi=0.8 \times 0.8$ jet) is used to capture the total energy of the $e^{+} e^{-}$pair. Figure 1(a) shows the efficiency of these two triggers as a function of the di-electron invariant mass.
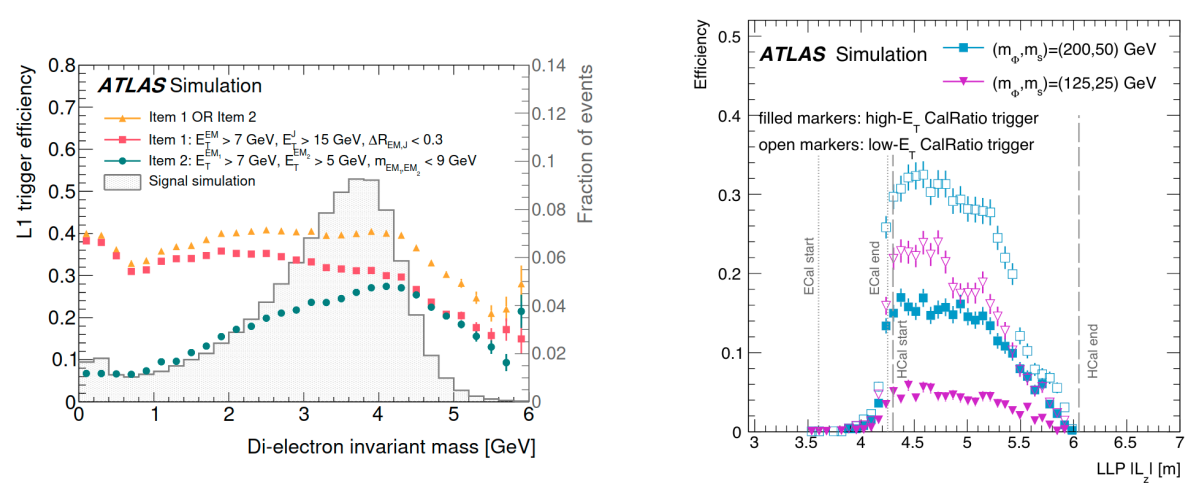

Figure 1: (a) Efficiency of L1Topo triggers developed for the $B^{0} \rightarrow K^{* 0} e^{+} e^{-}$measurement as a function of the offline invariant mass of electron pairs. The efficiency is determined using non-resonant simulated decays [2]. (b) Efficiency of the high- $E_{\mathrm{T}}$ non-topological CalRatio trigger (filled markers) and of the low- $E_{\mathrm{T}}$ topological CalRatio trigger (open markers) as a function of the absolute value of the LLP longitudinal decay position, $L_{z}$, for different mass hypotheses [2].

This type of triggers is also used to identify Higgs boson production and decays in the LHC. For example, the two jets that are generated from Higgs production via vector-boson fusion can be identified by looking at their position, angular separation and invariant mass. This procedure can enhance the signal acceptance of Higgs boson decays to invisible particles by about a $30 \%$ with respect to using only the non-topological missing transverse energy requirement.

Other analyses that benefit from L1Topo triggers are long-lived particle (LLP) searches. Some of them feature a heavy neutral scalar particle $\Phi$ decaying into LLPs which lead to displaced collimated jets with most of their energy deposited in the hadronic calorimeter. To target these events, a L1 trigger, called high- $E_{\mathrm{T}}$ CalRatio, was designed to select narrow jets with $E_{\mathrm{T}}>60 \mathrm{GeV}$. This cut strongly limits the efficiency of detecting signal events for models with $m_{\Phi}<200 \mathrm{GeV}$, but the $E_{\mathrm{T}}$ threshold can be lowered compensating the otherwise rate increase by using a dedicated topological trigger called low- $E_{\mathrm{T}}$ CalRatio. It uses tau TOBs to identify the energy deposits of these very collimated jets, given their smaller area. Since tau TOBs are reconstructed using both the electromagnetic and the hadronic calorimeters, one can filter the objects with high $E_{\mathrm{HAD}} / E_{\mathrm{EM}}$ by requiring that they do not overlap with any EM object above a certain energy threshold. Figure 
1(b) shows the efficiency of the high- $E_{\mathrm{T}}$ CalRatio and low- $E_{\mathrm{T}}$ CalRatio triggers as a function of the LLP longitudinal decay position. The topological selection recovers up to $20 \%$ of trigger efficiency for low- $m_{\Phi}$ hypotheses.

One last example of the multiple uses of L1Topo are the triggers for large-R jets. In this case, non-topological L1 triggers fail to capture the total energy of jets with a radius of $\Delta R>0.4$. Using the L1Topo simple cone algorithm, the total energy of these large- $\mathrm{R}$ jets is computed by combining the energy of all jets within a $\Delta R=1$ cone. Figure 2 shows how the simple cone algorithm can lower the energy threshold at which the trigger becomes fully efficient, particularly when the jet has a high sub-jet multiplicity.
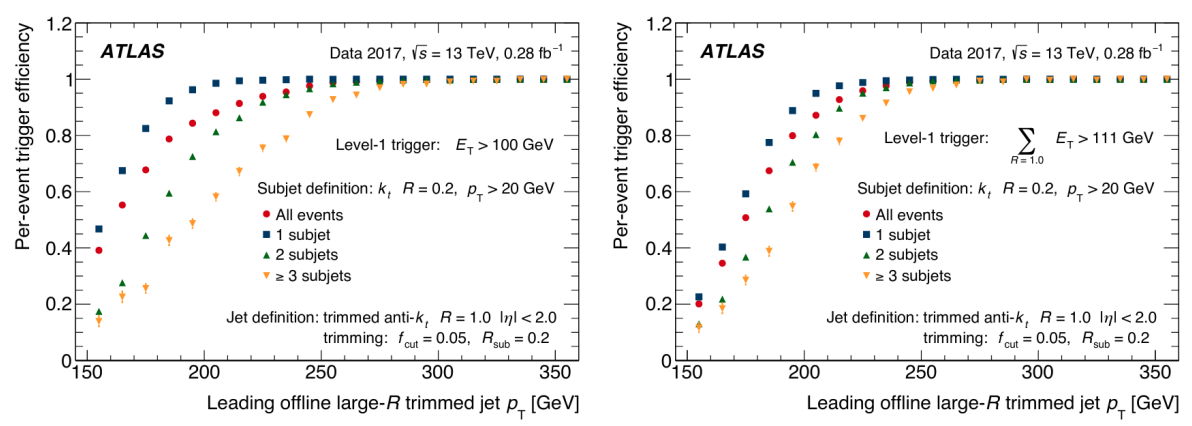

Figure 2: Efficiency curves for jet events with one, two or more than two sub-jets reconstructed using (a) the standard jet sliding-window algorithm and (b) the L1Topo simple cone algorithm [2].

\section{Phase-I L1Topo upgrade}

The ATLAS detector upgrade for Run 3 is referred to as Phase-I upgrade [3]. The Run 2 L1Calo cluster processor (CP) and jet energy processor (JEP) will be replaced by the electromagnetic feature extractor (eFEX) and jet feature extractor (jFEX), both providing higher granularity. The first module is designed to identify energy deposits corresponding to electrons, photons and tau particles, while the second performs jet, large-area tau, $E_{\mathrm{T}}^{\text {miss }}$ and $\sum E_{\mathrm{T}}$ trigger algorithms. In addition, a global feature extractor (gFEX) will be added to identify large-R jets using a coarser granularity, being also suitable to calculate quantities such as $E_{\mathrm{T}}^{\text {miss }}$ or $\sum E_{\mathrm{T}}$. Using the information from the new FEXes, L1Topo will be used to compute the multiplicity of the different L1Calo TOBs. The L1Muon endcap region will benefit from the New Small Wheel (NSW) detector, replacing the Run 2 inner stations of the endcap muon system, and will feature high-rate tolerance and improved resolution. In addition, an upgraded Muon to Central Trigger Processor Interface (MuCTPI) will provide full-granularity L1Muon information to L1Topo, which was not available in Run 2.

The new L1Topo system will consist of three modules with two processor FPGAs each (XilinxUltrascale+ XCVU9P). Two of the three modules will be used for topological selections and the remaining one, for L1Calo multiplicity triggers. During the beginning of Run 3, the legacy L1Topo and L1Calo systems will be used for triggering while the new systems are under commissioning. 


\section{References}

[1] ATLAS Collaboration, The ATLAS Experiment at the CERN Large Hadron Collider, JINST 3 (2008) S08003, URL: https: //cds . cern. ch/record/1129811

[2] ATLAS Collaboration, Performance of the ATLAS Level-1 topological trigger in Run 2, CERN-EP-2021-040, 2021, URL: https://cds . cern.ch/record/2765768.

[3] ATLAS Collaboration, Technical Design Report for the Phase-I Upgrade of the ATLAS TDAQ System, CERN-LHCC-2013-018, ATLAS-TDR-023, 2013, URL: https://cds.cern.ch/ record/1602235. 